\title{
Modelos de enseñanza y modelos de comunicación en las clases de ciencias naturales
}

\author{
Laura Levin* \\ Araceli Marcela Ramos* \\ Agustín Adúriz-Bravo**
}

Artículo recibido: 26-06-2006 y aprobado: 5-08-2008

Teaching models and models of communication in science classes

Resumen: El objetivo de la investigación relatada en este trabajo fue analizar si el estilo de enseñanza al que dice adherir el profesorado de ciencias naturales se expresa en las diversas formas de comunicación que se establecen en sus clases. El foco de la observación de clases estuvo principalmente en las interacciones lingüísticas entre profesor/a y estudiantes. La enseñanza y la comunicación se tipificaron con diversos modelos teóricos, que aquí se revisan. A partir del análisis de las clases observadas se robustecen las siguientes precisiones: a) el modelo "didáctico" constructivista necesita en algún momento del despliegue efectivo de una comunicación dialógica en el aula; b) por tanto, "adherir" declarativamente al constructivismo mientras se generan modos de comunicación clausurantes en clase deja al descubierto modelos implícitos de transmisión o de condicionamiento c) sin embargo, en las aulas argentinas se observan tipos híbridos de práctica en donde el lenguaje asume muchos papeles.

Palabras clave: Modelos de enseñanza, modelos de comunicación, interacción en el aula, lenguaje científico escolar, constructivismo, observación de clases.
Abstract: The aim of this study was to analyse whether science teachers' style is expressed in the different communication schemes established in their classes. Classroom observation was focused on linguistic interactions established between teacher and students. Teaching and communication were characterized by means of several theoretical models, which are discussed here. From the classroom observation analysis, it was possible to draw the following statements: 1 . the constructivist teaching model requires, at least in some periods of the time, the effective use of dialogic communication inside the classroom; 2Therefore, the declarative 'adherence' to constructivism while 'closing' communication models are generated in the classroom reveals implicit models of transmission or conditioning; 3 . However, it is possible to observe that, in Argentinean classrooms there are hybrid types of practice in which language plays different roles.

Key words: Teaching models, communication models, classroom interaction, school scientific language, constructivism, and classroom observation.

\footnotetext{
* Departamento de Biodiversidad y Biología Experimental, BBE. lale@bg.fcen.uba.ar

** Universidad de Buenos Aires, UBA. Facultad de Ciencias Exactas y Naturales, FCEyN.

andurizbravo@yahoo.com.ar.
} 


\section{Introducción}

Hoy en día, uno de los problemas fundamentales de la innovación en educación es encontrar formas de comprometer a las aulas para crear y mantener condiciones en las que pueda prosperar un diálogo abarcador, democrático y abierto durante la enseñanza (Sarramona, 1988; Burbules, 1999; Mortimer y Scott, 2003; Rice, 2005; Sagastizábal, 2006). De allí la importancia que ha cobrado, en el campo de la investigación educativa, el estudio de las formas y estilos que asume la comunicación al enseñar (Contreras, 1990; Sierra, 2000). El problema de la comunicación significativa (dialógica) de contenidos disciplinares en las aulas de ciencias naturales también viene siendo identificado como una línea de trabajo central para la didáctica de las ciencias (Lemke, 1997, 2002; Sanmartí, 2002, 2003; Márquez et al., 2003, 2006; Mortimer y Scott, 2003; Izquierdo et al., 2004, 2007; Izquierdo, 2005; Marbà y Márquez, 2006; Marbà et al., 2006; Scott et al., 2006; Scott y Ametller, 2007).

José Contreras (1990) define el proceso de enseñanza como un proceso de comunicación humana que reúne cinco características: es intencional, forzado, jerárquico, grupal e institucionalizado. En resumen, la enseñanza se diseña siguiendo finalidades claramente definidas, se da en un contexto normativo en el seno de un sistema de relaciones sociales, esta mediada por un lenguaje que, entre otras muchas cosas, comunica contenidos disciplinares específicos, y se desarrolla constreñida por el marco de sistemas altamente formalizados creados para el efecto.

En este trabajo nos interesa realizar un primer acercamiento a las relacio- nes de convergencia o de contradicción que se establecen entre las formas de enseñar y las formas de gestionar la comunicación en clases reales de ciencias naturales. Para el análisis de nuestro corpus de datos, recuperaremos dos tipologías muy genéricas: una de modelos comunicativos y otra de modelos "didácticos" (de enseñanza). También queremos examinar más detalladamente algunas de las características de la comunicación didáctica que se genera en las clases de ciencias, para lo cual recurriremos a diversas aportaciones teóricas provenientes de la pedagogía, de la didáctica de las ciencias naturales y de los nuevos estudios sobre el lenguaje en el aula.

\section{Marcos teóricos}

En esta sección presentaremos dos marcos teóricos clásicos, "instalados" en forma relativamente amplia dentro de la comunidad académica iberoamericana de investigación educacional, que fueron diseñados para clasificar los procesos de comunicación y de enseñanza mediante analogías o tipificaciones taxonómicas y "modelizadoras" (es decir, de representación teórica). Por cierto, entendemos que estos marcos no son los únicos posibles para el análisis, pero, funcionando a modo de "lentes conceptuales" muy potentes, a nosotros nos permiten encontrar cruces significativos en el estudio etnográfico de nuestras aulas de ciencias naturales (Galagovsky et al., 1998).

El primer marco teórico proviene de la pedagogía, y caracteriza la comunicación "didáctica" (esto es, aquella dirigida intencionalmente a la enseñanza) con tres metáforas, según el grado de participación de los actores (profesorado 
y estudiantado) en la co-construcción del discurso de aula. El segundo marco proviene de la didáctica de las ciencias de línea francesa, y tipifica las "formas de enseñar" según la imagen de aprendizaje y de estudiante que el profesorado sustenta para organizar su práctica (por ello, esta clasificación "sintoniza" con fuentes psicológicas y cognitivas).

En el caso de este segundo marco de análisis, hablamos de modelos didácticos (es decir, modelos de enseñanza) sabiendo que esta expresión es problemática por su multiplicidad de sentidos (AdúrizBravo, 2001; Galagovsky y Adúriz-Bravo, 2001). La decisión ha sido respetar aquí la nomenclatura original postulada por Jean-Pierre Astolfi (entrecomillándola, para señalar su problematicidad), pero en la subsección correspondiente haremos un breve paréntesis para discutir algunas dificultades que tiene esta nomenclatura'.

\section{Los modelos comunicativos}

El pedagogo español José Contreras Domingo (1990) plantea tres modelos generales apoyados en sendas analogías para conceptualizar el proceso de comunicación didáctica en la escuela:

1. El modelo telegráfico, que entiende la comunicación como un proceso de transmisión lineal y unidireccional de información entre un emisor (profesor/a) y unos receptores (estudiantes). Todo lo que acontece alrededor no importa para el proceso, y son solo "ruidos" que interfieren con la comunicación eficaz del mensaje.

1 Agradecemos a un evaluador anónimo de nuestro artículo por sus sugerencias en la línea de añadir esta discusión, que consideramos valiosa para los lectores.
2. El modelo orquestal. Para este modelo, la comunicación es un proceso social donde todos participan de alguna forma más o menos limitada. La analogía es una orquesta donde todos tocan la misma partitura, no ya un intérprete (profesor/a) y su auditorio (estudiantes), como en el modelo anterior. Lo que ocurre alrededor es también parte del proceso. La comunicación se establece no sólo a través de la palabra, sino también a través de los gestos. Sigue habiendo un director de orquesta (profesor/a), y los mensajes (contenidos) son bastante unívocos por su naturaleza externa, normativa e institucionalizada.

3. El modelo sistémico, que entiende la comunicación como un sistema complejo de elementos interactuantes, y analiza esos elementos y las múltiples y diversas relaciones que se establecen entre ellos. El factor crítico en la comunicación no es, como en el modelo anterior, el emisor (músico) o la naturaleza del mensaje (partitura), sino más bien la capacidad de los sucesivos receptores para decodificar y dar sentido a lo que los emisores dicen o hacen. Deben existir un código lingüístico y un acervo cultural compartidos. Emisores y receptores intercambian mensajes por distintos canales (verbal, gráfico, gestual) y con distintos códigos, no siempre convergentes. Los mensajes tienen un contenido informativo y un contenido relacional, esto es, dicen sobre la relación que se establece entre los comunicantes (este es, para el psicoterapeuta y semiólogo austriaco Paul Watzlawick, uno de los axiomas de la comunicación: Watzlawick et 
ál., 1981). El mensaje que recibe el receptor es creación tanto del emisor como de él mismo: los mensajes son entonces elaboraciones complejas. Profesor/a y estudiantes participando en la comunicación son simultáneamente emisores y receptores.

El modelo sistémico tiene en cuenta para el análisis que, debido al carácter equívoco de la comunicación humana, los interlocutores necesitan desplegar procedimientos de reconocimiento de la comprensión correcta, llamados con el nombre genérico de realimentación. El uso de códigos que son no solo lingüísticos, sino también de relación social, debe dominarse para tomar parte eficientemente en la comunicación. El dominio de esos códigos se adquiere participando de los sistemas culturales donde ellos tienen vigencia. En este sentido, las ciencias naturales, como sistema cultural, poseen códigos propios y el acceso a ellos debe plantearse desde la educación formal (Lemke, 1997, 2002).

El medio escolar supone un contexto diferenciado con sus propios códigos, con su cultura peculiar, que es necesario conocer para participar adecuadamente en él. Existe además una comunicación mediada, en la cual los objetos y las instituciones sociales comunican. La escuela como institución comunica, y aquello que se comunica en la educación y en la enseñanza variará de acuerdo al modelo educativo que ella sustente. En la comunicación didáctica se ponen en juego muchos elementos; nos interesa señalar los siguientes, siguiendo a Contreras (1990):

1. Las fuentes de información (emisores, textos, materiales, etc.), reguladas por el profesor/a.
2. El mensaje. Además del conocimiento académico (currículo formal), el profesor/a pone en juego otros mensajes: sobre las formas de participación adecuadas (relaciones sociales), sobre las actividades que tienen que realizar los estudiantes con los contenidos de la enseñanza. Resulta por ello imposible separar qué se enseña de cómo se enseña.

3. Las reglas de participación. Las clases son ambientes comunicativos diferenciados con reglas propias acerca de cómo y cuándo participar, que además están afectados por la escuela y el sistema educativo, y por el marco más general de la sociedad y la cultura de un pueblo.

A estos tres elementos genéricos de la comunicación en el aula para la enseñanza de los contenidos curriculares, hay que añadir los constreñimientos específicos que las ciencias naturales como acervo cultural imponen. En este sentido, hay especificidades propias de la comunicación científica que han de ser tenidas en cuenta en la labor del profesorado de ciencias (Izquierdo et ál., 2004, 2007; Izquierdo, 2005; Marbà y Márquez, 2006).

Nicholas Burbules (1999), en un texto ya clásico, plantea la idea de la enseñanza como diálogo, entendiendo el diálogo como una forma de comunicación pedagógica. El diálogo es una relación comunicativa "simbiótica" entre pares, que exige un compromiso cognitivo y también emocional. En sus planteos teóricos, este autor distingue entre preguntas auténticas o divergentes y preguntas aparentes o convergentes. Las preguntas auténticas quedan fundamentalmente abiertas, ya que su respuesta 
no está preestablecida; las aparentes (pseudopreguntas), en cambio, delimitan artificialmente lo que puede valer como una respuesta satisfactoria, se dirigen a una respuesta específica.

Las pseudopreguntas comunican mensajes implícitos sobre las respuestas deseables (fijadas por el saber del profesor/a), y apelan a la ingenuidad de los estudiantes. En este marco rara vez ellos cuestionan el sentido de la comunicación o introducen preguntas que salven el abismo entre su marco de referencia y el de los profesores (Galagovsky et ál., 1998). Este escenario de enseñanza-ficción (Cañal y Porlán, 1987) no es solo responsabilidad del profesorado, sino que las distintas influencias institucionales, como las presiones por cubrir los contenidos y las evaluaciones (basadas en pruebas) del rendimiento de los estudiantes, del profesorado y de la escuelas, ponen a la clase en esta situación.

Burbules (1999) sostiene también que el éxito del diálogo didáctico debería evaluarse por la naturaleza del intercambio comunicativo que se desenvuelve en la relación dialógica, no sólo por sus resultados. A la inversa, el fracaso en la comunicación didáctica viene dado por aquello que interrumpe abruptamente la discusión, que determina de antemano ciertas áreas de indagación o que acalla o ahoga ciertos puntos de vista. La situación escolar es muchas veces antidialógica, al igual que gran parte de nuestra sociedad. Para que exista un auténtico juego dialógico debe existir participación, compromiso y reciprocidad entre los participantes (Mortimer y Scott, 2003; Scott et ál., 2006). En este contexto, virtudes como la tolerancia o la paciencia son indispensables.
Diversas instituciones sociales propenden a reforzar ciertas formas de pensar y a desalentar otras; por ejemplo, los sistemas sociales de poder y de privilegio tienden a reforzar las asimetrías en el diálogo viciando la reciprocidad y el respeto que este exige. Las experiencias de castigo o intimidación se dirigen a silenciar "determinadas voces, en ámbitos incluso aparentemente abiertos" (Burbules, 1999: 203). Si la educación muestra las respuestas correctas, se suprime la preferencia por discusiones más interesadas en la indagación, la construcción y los puntos de vista divergentes, estrategias que están en la base de la didáctica de las ciencias actual. Este estilo comunicativo antidialógico, o autoritario (Mortimer y Scott, 2003), tiene una incidencia negativa en la calidad de la educación científica, pues entra en abierta contradicción con la propia naturaleza de la ciencia (Jiménez Aleixandre, 1996; Duschl, 1998).

El currículo de ciencias tradicional no reconoce la necesidad del desarrollo de aptitudes comunicativas (Sanmartí, 2003); incluso, en ciertas situaciones, poner de manifiesto virtudes comunicativas puede resultar inseguro, porque entra en contradicción con el modelo de ciencia detentado, que es en cierto sentido a-retórico en tanto que apodíctico o “autoevidente” (Izquierdo, 2005; Marbà et ál., 2006).

El currículo se centra en cubrir el contenido; las metas educativas se evalúan mediante pruebas en las cuales los resultados que no pueden "medirse" son relegados a una posición subordinada. Existe una concepción que centra el papel del profesor/a en el manejo (control) del aula; el mantenimiento de las condiciones de orden y disciplina se convierte 
en un fin en sí mismo. Esta concepción técnica de la didáctica tiende a orientar los comentarios de profesorado y estudiantes hacia un habla de control. Cuando los estudiantes introducen preguntas o se apartan del tema, los docentes que adhieren a este modelo ven en ello una prueba de que han perdido el control e inician acciones para restituir el orden.

\section{Los modelos "didácticos"}

Como se adelantó, la expresión de 'modelo didáctico' tiene múltiples significados (ver una discusión en AdúrizBravo, 2001); este hecho puede tornar problemático su uso en el campo de la didáctica de las ciencias naturales. A los fines de la discusión que sigue, conviene distinguir al menos tres sentidos claramente divergentes de tal expresión:

1. A veces se llama 'modelo didáctico', desde un punto de vista que recupera la etimología de la palabra (en griego clásico, didaskein es 'enseñar'), a un modelo de enseñanza, es decir, a la configuración praxiológica "ideal" (teórica) de un profesor-tipo que planifica y ejecuta su acción de aula desde una determinada mirada paradigmática (conductista, constructivista, etc.) y, por tanto, homogénea. La didáctica francesa, posicionada "desde el punto de vista del proceso de enseñanza-aprendizaje” (Porlán y Rivero, 2001: 221; la traducción es nuestra), recupera este primer sentido de la terminología de modèle didacti$q u e$, como es el caso del texto de Astolfi que utilizaremos como insumo principal para nuestro análisis.

2. Otras veces, se reserva la expresión de 'modelo didáctico' para designar -de forma abreviada- un modelo teórico (en el sentido de la visión basada en modelos: Develaki, 2007) perteneciente a la didáctica de las ciencias naturales como disciplina científica (ver Izquierdo y Adúriz-Bravo, 2003). En este segundo sentido, el modelo de cambio conceptual o el modelo de ciencia escolar serían modelos didácticos muy conocidos.

3. Por último, y de forma cada vez más frecuente, se emplea el término 'modelo didáctico' para referir a un modelo teórico de las ciencias naturales cuando se lo ha transpuesto para el aula, o a una re-representación analógica de ese modelo que auxilia en la enseñanza (ver Galagovsky y Adúriz-Bravo, 2001). En este sentido, los modelos científicos escolares y los modelos analógicos serían modelos didácticos.

Es importante señalar que, en estos tres casos discutidos, la elección de la palabra 'modelo' para referirse respectivamente a una forma de dar clases, a un postulado disciplinar novedoso propuesto por investigadores o a un contenido de enseñanza y sus análogos no es arbitraria ni trivial. En las tres expresiones arriba reseñadas se quiere indicar una cierta forma "fuerte" de concebir las entidades bajo estudio. No se trata sólo de conductas, ideas o contenidos, sino de estructuraciones simbólicas de todos ellos que cumplen con los dos requisitos actualmente aceptados para que algo sea considerado un "modelo"2:

1. de alguna manera "capturan" lo esencial y nuclear de esas entidades,

2 Seguimos aquí la vasta literatura reciente acerca de los modelos teóricos que circula dentro de nuestra disciplina (ver Adúriz-Bravo, 2001; Izquierdo y Adúriz-Bravo, 2003; Erduran y Duschl, 2004; Gallego Badillo, 2004; Develaki, 2007). 
es decir, son reconstrucciones teóricas; $\mathrm{y}$

2. de alguna manera "guían" la intervención sobre la realidad proveyendo de reglas paradigmáticas para la acción.

Ahora bien, según cómo el profesor/a conciba desde el punto de vista teórico la enseñanza de su área específica de conocimiento, lleva adelante una manera particular de gestionar su trabajo en el aula, que llamaremos, según la primera de las acepciones discutidas más arriba, modelo "didáctico”. Esta gestión "modélica” del aula, naturalmente, incluye el establecimiento de modos y estilos de comunicación. Astolfi (1997) diferencia tres modelos "didácticos" principales:

1. El modelo de transmisión. El conocimiento sería un contenido de enseñanza que se imprimiría en la cabeza de los estudiantes (el "contenedor") como en una cera blanda (analogía de la tábula rasa). Aquí la enseñanza se vale de un esquema de comunicación vertical emisor-receptor (análogo al modelo telegráfico), derivado de la teoría de la información. En este modelo el estudiante es un sujeto pasivo, del cual se espera que ponga atención, sea regular en el trabajo y en el esfuerzo y dé pruebas de voluntad para no quedarse atrás. Se trata de una "pedagogía de ideas claras": bastará con que el profesor explique claramente, comenzando desde el principio, avanzando progresivamente y citando buenos ejemplos, para que el conocimiento se transmita y se inscriba en la memoria de los estudiantes.

En este primer modelo, el fracaso y el error deben ser evitados. Si pese a todo se producen, resulta natural una sanción, puesto que su aparición sería responsabilidad del estudiante, quien no habría adoptado la actitud esperada. Este modelo didáctico puede ser adecuado frente a un público motivado e informado, que posee estructuras intelectuales similares a las del profesor/a (más allá de la asimetría de sus conocimientos disciplinarios), que dispone de elementos de conocimiento en el campo de aprendizaje en cuestión, y que aprovecha la exposición sistemática para organizar y reestructurar la información previa (Ausubel et ál., 1983).

2. El modelo de condicionamiento, desde la perspectiva conductista. Se considera al estudiante como un agente pasivo; se ignoran los procesos que ocurren al interior de la mente (analogía de la caja negra). El aprendizaje resulta de una serie de condicionamientos, se produce por asociación entre estímulos y respuestas y por el posterior refuerzo de las conductas correctas. Se juzga el comportamiento observable al final del aprendizaje (a modo de "salida", o output, de la caja negra). Se trata de una "pedagogía del éxito", puesto que trata de encontrar los medios eficaces para evitar el error. Los contenidos se dividen en pequeñas unidades, cada una de las cuales se apoya sobre la anterior (estructuración del currículo por objetivos). Si a pesar de ello el error persiste, no es ya responsabilidad del estudiante sino del profesor, o más bien del programa. El error se desconoce como fuente de aprendizaje. Este modelo, a pesar de sus limita- 
ciones, suele aplicarse para construir la evaluación, entendiéndola como momento de recogida sistemática de información sobre los logros.

3. El modelo constructivista. Esta perspectiva considera al estudiante como un agente activo que construye significados en respuesta a la situación educativa. Reconoce la existencia de la mente y sus procesos. Niega que el estudiante absorba pasivamente la información suministrada por el profesor/a o depositada en los textos. El error ya no es considerado una deficiencia del estudiante ni una falla del profesorado. No se castiga ni evita, se busca como fuente de aprendizaje y de reflexión dentro de un sistema que privilegia la metacognición. El modelo constructivista requiere el establecimiento de formas de comunicación dialógicas interactivas (Mortimer y Scott, 2003) en al menos algunos momentos de la enseñanza. En el caso específico de la enseñanza de las ciencias naturales, el modelo constructivista pone al "hablar y escribir ciencias" como uno de los pilares de una genuina actividad científica escolar (Izquierdo et ál., 2004).

\section{Objetivo e hipótesis de partida}

El objetivo del presente trabajo fue analizar de qué manera las formas de enseñar a las que dicen adherir algunos profesores/as de ciencias naturales y aquellas que efectivamente se ponen en juego en sus prácticas se relacionan con los esquemas de comunicación que ellos son capaces de establecer en sus aulas, y cómo estos apoyan u obturan la posibilidad de una enseñanza construc- tivista dialógica. Las formas de enseñar se miran desde la tipología de modelos "didácticos" de Astolfi; los esquemas de comunicación, desde la tipología de modelos comunicativos de Contreras. Además, se hace un análisis de los registros de comunicación de aula con las categorías teóricas discutidas más arriba.

Desde la teoría, existe una fuerte correlación entre modelos "didácticos" y modelos comunicativos: la enseñanza transmisiva se expresa en una comunicación mayormente telegráfica que tiende a minimizar los ruidos, y la enseñanza constructivista requiere de una comunicación sistémica en donde se ponga el acento en dar sentido a lo que se hace, dice y piensa. Sin embargo, estos modelos teóricos "puros" no se encuentran como tales en el profesorado de ciencias naturales. La práctica profesional de un profesor excede los mandatos de su propia teoría implícita, y esta además no es homogénea (Porlán y Rivero, 1998, 2001). Por tanto, partimos de la hipótesis de que en las aulas de ciencias naturales pueden darse formas de enseñanza y de comunicación híbridas y entrecruzamientos entre los elementos característicos de cada modelo teórico. Nuestro objetivo es ver si la voluntad de un profesor/a de ciencias naturales de adherir a una forma de enseñanza queda respaldada por su capacidad de establecer esquemas de comunicación coherentes con ella en momentos específicos de sus clases.

\section{Metodología empleada}

Los datos para nuestro análisis fueron principalmente obtenidos por medio de observaciones de clase. Se observaron cinco profesoras de ciencias naturales 
con diversidad de formaciones iniciales y de experiencias profesionales. El foco de la observación estuvo principalmente en las interacciones entre profesorado y estudiantes dentro del aula en clases de ciencias naturales.

Si bien se registraron también fuera del contexto de las clases algunos otros elementos que podrían resultar relevantes para la investigación (condición socioeconómica de los estudiantes, características edilicias de los establecimientos educativos, cartelería con mensajes, clima institucional, documentación, producciones escritas, etc.), ellos no serán analizados aquí. Se realizaron además entrevistas informales después de las clases a profesoras y estudiantes, a fin de comentar el desarrollo de las mismas, y entrevistas a las autoridades para obtener más datos sobre las instituciones. Estos datos, al igual que los anteriores, sirvieron para reforzar nuestras interpretaciones y "triangularlas", pero no son objeto de discusión en este trabajo $^{3}$. Para resumir las condiciones de contexto de nuestra investigación, la tabla 1 expone algunas de las características de la muestra estudiada.

\begin{tabular}{clcl}
\hline Clase & \multicolumn{1}{c}{ Institución y características } & Profesora & \multicolumn{1}{c}{ Asignatura y nivel } \\
\hline C1 & I1: secundario público & P1 & Biología de 3er año (15-16 años) \\
\hline C2 & I1 & P1 & Biología de 2 año (14-15 años) \\
\hline C3 & 12: secundario privado confesional & P2 & Biología de $4^{\circ}$ año (16-17 años) \\
\hline C4 & I2 & P2 & Biología de 4 año \\
\hline C6 & 13: secundario privado laico & P3 & Biología de 3er año \\
\hline C7 & 14: secundario dependiente de la & P3 & Biología de 1er año (13-14 años) \\
\hline universidad & P4 & Biología de 3er año \\
\hline C9 & 14: secundario privado confesional & P3 & $\begin{array}{l}\text { Educación para la salud, } 5^{\circ} \text { año } \\
\text { (17-18 años) }\end{array}$ \\
\hline
\end{tabular}

Tabla 1. Muestra de clases observadas.

\section{Resultados y discusión \\ Caso 1}

Las observaciones $\mathrm{C} 1$ y $\mathrm{C} 2$ se realizaron en la misma institución (ver tabla 1), en dos clases dictadas por la misma profesora (P1), con dos grupos de estudiantes diferentes. Durante la clase C1, en el laboratorio, los estudiantes realizaron observaciones de distintos tipos celulares al microscopio, basándose en una guía de trabajos prácticos. En la clase C2 trabajaron en el aula sobre una guía de investigación acerca de la estructura celular; la guía había sido elaborada previamente fuera de clase utilizando distintos textos.

3 El análisis empírico completo, incluyendo aquellos elementos que aquí no se discuten, se puede consultar en Levin et ál. (2004). 
Analizando lo observado en el aula desde el punto de vista del modelo "didáctico", la profesora P1 se inscribiría sin grandes problemas en el modelo de transmisión: en este modelo el estudiante es considerado un sujeto pasivo, del cual se espera que ponga atención. Para esta profesora, el fracaso y el error deberían ser evitados: esto se evidencia en las reiteradas veces en que ella utiliza las frases "ya te lo dije", "ya les enseñé" y otras por el estilo. La falta de tolerancia que muestran estos intercambios condiciona la posibilidad de un diálogo genuino cercano al del modelo sistémico; ese diálogo, según Burbules (1999), exigiría, además del compromiso cognitivo, un compromiso emocional (interés, confianza, respeto, aprecio, tolerancia, apertura, disposición a escuchar).

En el modelo de transmisión, el estudiante absorbe pasivamente la información comunicada por la profesora y por los materiales escritos; no hay genuina construcción de conocimiento. Esto se evidencia en ambas clases, pero particularmente en la clase $\mathrm{C} 2$, donde los estudiantes "recitan" la información extraída de los libros. Por ejemplo:

Profesora (P1): Bueno, falta la última pregunta. A ver [nombrando a un estudiante]: ¿qué color, forma, tamaño, tienen las células?

Estudiante (E): Las células son como globos que presentan grandes variedades. P1: No, está mal.

E: ¡Lo copiamos del libro!

Aquí el aprendizaje se desarrolla en su mayoría dentro de un esquema de comunicación unidireccional desde el emisor hacia el receptor, según el modelo telegráfico de Contreras (1990). En este modelo aparecen sólo preguntas conver- gentes que se dirigen a una respuesta específica y por tanto, delimitan artificialmente lo que puede valer como un diálogo satisfactorio (Burbules, 1990). Se privilegia, además, la respuesta correcta (P1: jÉsa es la observación correcta!). El error, como no es visto como una fuente de aprendizaje, es sancionado fuertemente. Por ejemplo:

\section{E: Profe, ¿está bien?}

P1: Así no era. ¡Usted haga lo que quiera, que yo después me llevo el práctico [para corregirlo]!

Según Burbules (1990), experiencias de castigo o intimidación como la anterior llegan a silenciar las voces de quienes tienen menos poder en ámbitos que aparentan ser abiertos, como la escuela. Otro ejemplo de intimidación es:

P1: ¡Hay gente que no viene más al laboratorio!

E: ¿Quién?

[La profesora no responde.]

Dentro de este modelo didáctico, la profesora trabaja con los estudiantes que "responden" y no cometen errores, de acuerdo con lo que Perkins (1995) llama la teoría del rendimiento, que privilegia la capacidad: se piensa que el aprendizaje depende fundamentalmente de la inteligencia de las personas y no de su esfuerzo. Esta concepción beneficia a unos pocos estudiantes y perjudica al resto. La actitud de la profesora hacia quienes ella ve como más dotados les infunde confianza en sí mismos, y aprenden más porque se espera más de ellos. Los "menos dotados", mientras tanto, quedan relegados de los intercambios comunicativos.

Según Alicia Camilloni (1998), cuando un profesor califica a un estudiante 
es frecuente que recurra a apreciaciones personales. En este caso, por ejemplo:

P1: [A un estudiante.] No se pinta todo el campo, no haga como [otro estudiante, señalado con el dedo, que, angustiado, pintó todo el campo]. ¡Así no!

En este ejemplo aparece el efecto de halo, que se manifiesta en la predisposición a calificar positivamente las nuevas realizaciones de los buenos estudiantes o a la inversa con los tenidos como malos. Este efecto actúa como un prejuicio que impide una consideración más objetiva y justa del rendimiento.

David Perkins (1995) postula el modelo de la búsqueda trivial, según el cual el aprendizaje consiste en la mera acumulación de hechos y rutinas estereotipadas. Este tipo de aprendizaje se evidenciaría en la clase $\mathrm{C} 2$, donde se ve un uso excesivo de términos y definiciones científicas vaciadas de contenido: este fenómeno es lo que se denominó nominalización didáctica (Galagovsky et ál., 1998). Pero si el estudiante no tiene la posibilidad de aplicar el nuevo conocimiento para lograr objetivos específicos, a menudo aprende hechos que sólo se pueden recordar en contextos limitados, y que, en toda otra situación quedan inertes. Los estudios muestran que la información que es meramente memorizada permanece inerte aunque sea relevante en situaciones nuevas (Bransford y Vye, 1989).

\section{Caso 2}

Las observaciones C3 y C4 se realizaron en una segunda institución, en dos clases impartidas por la misma profesora (P2) y con el mismo grupo de estudiantes (ver tabla 1). Durante la clase C3, los estudiantes realizaron en el laboratorio un trabajo práctico (titulado "Reconocimiento de sustancias orgánicas”) basándose en una guía. Al día siguiente (clase C4), en el aula, a fin de analizar la experiencia discutieron las preguntas incluidas en esa misma guía.

Consideramos que esta profesora, al igual que P1, se inscribe en un modelo "didáctico" de transmisión. En coherencia con esto, el esquema de comunicación que ella deja prosperar en sus clases responde fuertemente al modelo telegráfico. Se supone que el estudiante sólo ha de recibir la información; se demanda su participación mayormente a través de preguntas convergentes:

P2: Voy a hacer una pregunta. ¿Quién me responde levantando la mano por la positiva? Tengo un tubo de ensayo con solución de almidón. ¿Qué sucede si escupo en el tubo, y luego agrego reactivo de Fehling? Levanten la mano y contesten correctamente. [El énfasis es nuestro.] E: Se degrada con la amilasa salival. P2: ¿Qué pasa con el Fehling si el almidón se degradó parcialmente con la amilasa salival? [Silencio, nadie contesta.] Bueno, quiero que me contesten correctamente. [El énfasis es nuestro.] Lo piensan para mañana.

Cuando hay diversidad de respuestas, la profesora generalmente presta atención a las correctas, ampliándolas, acotando algo y exclamando frecuentemente: “ibien!”, “¡correcto!”, “iperfecto!”. Las respuestas incorrectas son desoídas o aparece alguna recriminación. Esto sabotea el establecimiento de una construcción colectiva de significados.

A diferencia de lo que vimos en las clases de P1, donde las fallas en el diálogo parecían deberse a una falta de compromiso emocional (sobre todo al 
desinterés), en este caso la falla en la comunicación aparece más fuertemente ligada a la concepción de la tarea del profesor centrada en el control: la "disciplina" (buena conducta) se convierte en un fin en sí mismo (Galagovsky et ál., 1998; Burbules, 1999), avalado desde la institución. Como nos refiere la profesora P2 en la entrevista posterior: "La institución se ocupa de la disciplina, que es rigurosa. Las autoridades circulan por la escuela y hay un control estricto de lo que sucede en la misma. Los directores entran frecuentemente a la clase para ejercer el control personalmente".

La educación no es solo lo que ocurre en el aula; las prescripciones curriculares, los modos de organización de la escuela, las condiciones físicas y materiales también influyen sobre el currículo. Y todas estas variables constituyen las conexiones del aula con las estructuras sociales. Cualquier intento de actuación del profesorado tiene que vérselas con todos estos aspectos (Apple, 1979). La profesora P2 se muestra conforme con la alta injerencia institucional y en algún momento acota que, de este modo, "no tengo que ocuparme de la disciplina y sé que voy a poder terminar con el tema planificado".

El currículo de ciencias puesto en marcha por la profesora P2 se centra en cubrir el contenido, que se evalúa mediante pruebas escritas (Burbules, 1999). La prueba se utiliza como medio de control:

P2: ¡Silencio! Hablamos sobre la prueba que van a dar ustedes. A mí no me interesa la prueba, yo vengo y la tomo.

Aunque en la clase predominan las preguntas convergentes, aparece también alguna pregunta divergente, señal de la existencia de un posible modelo didáctico híbrido:

P2: ¿Qué uso se les ocurre que pueden tener en la vida cotidiana los reactivos que se utilizan para identificar sustancias orgánicas?

\section{Caso 3}

Las observaciones C5 y C6 se realizaron en una tercera institución, en dos clases de biología dictadas por la misma profesora (P3) con dos grupos de estudiantes diferentes (ver tabla 1). En la primera clase (C5), luego de la proyección de un video sobre el tema de los neurotransmisores, explicado y evaluado en clases previas, la profesora entregó un artículo periodístico sobre el cual los estudiantes debían realizar un trabajo escrito que incluía ponerle el título más adecuado y hacer un cuadro sinóptico o mapa conceptual a partir del mismo. Los estudiantes trabajaban con autonomía resolviendo en cada grupo las consignas. La profesora circulaba por los grupos, los estudiantes preguntaban con libertad; el diálogo era fluido y el clima distendido.

En la segunda clase (C6), los estudiantes trabajaron sobre un cartel que reunía las ideas previas que habían surgido sobre el tema de célula en clases anteriores, a partir de la consigna de "anotar las cosas que conocían sobre la célula". El trabajo de la clase era "ampliar la información sobre las células". A partir del cartel, la profesora fue leyendo y trabajando "las cosas que ' 1 er Año' conoce sobre la célula".

Por otro lado, se observó a la misma profesora P3 en otra clase (C9) en una institución diferente, en la asignatura de educación para la salud. En esta clase se 
realizó una puesta en común sobre la enfermedad de Chagas-Mazza (tripanosomiasis americana). Se trató de una clase de cierre luego de la información teórica brindada por la profesora. La actividad en grupos por parte de los estudiantes incluía: encuestas a personas que hubieran vivido en alguna provincia del interior de la Argentina, la proyección de la película argentina Casas de Fuego (sobre la vida del médico Salvador Mazza, estudioso de la enfermedad) y la elaboración de un trabajo escrito, en grupos, en el cual se ligaban escenas de la película con los contenidos teóricos y con lo relevado en las encuestas. Sentándose en ronda, cada estudiante, voluntariamente y por turno, compartió con el grupo y con la profesora sus vivencias, sentimientos y dudas generados por el tema. La discusión giró sobre la falta de difusión, en los medios de comunicación, de los alcances de la enfermedad, que no es tomada en cuenta como una problemática sanitaria general en nuestro país.

Estas tres observaciones nos llevan a tipificar que en las clases de esta profesora se establece un esquema de comunicación explicable con el modelo sistémico (Contreras, 1990). La profesora P3 está atenta a la capacidad de los estudiantes para decodificar lo que ella va diciendo o haciendo; a su vez, se dota de sentido colectivo a las intervenciones de los estudiantes. Los mensajes se intercambian mediante distintos recursos semióticos (habla, gesto, imagen fija y móvil, texto) y con distintos códigos; no siempre el uso de los lenguajes es convergente (Márquez et ál., 2003, 2007). El uso de códigos de relación social muestra el dominio que la profesora P3 tiene de la situación co- municativa. Ella hace uso de numerosos recursos que invitan a los estudiantes al diálogo; entre estos recursos apareció frecuentemente el humor:

P3: Levanten la mano [aquellos] a los que les suene [a] chino.

P3: Otro tema, próximamente en la misma sala.

Sin embargo, a veces se cae en la ironía y se evidencia la asimetría de poder (Galagovsky et ál., 1998):

P3: Aquí largaron con las palabras del [juego del] ahorcado.

Como adelantamos, la profesora P3 toma en todo momento lo que los estudiantes dicen, dando lugar a que todos se expresen, permitiendo la elaboración de mensajes complejos producto de todos los actores que participan de la situación comunicativa:

E: [Mostrando una foto.] La célula, ¿es lo rosa o lo celeste?

P3: La célula es la totalidad. ¡Bien lo que preguntás, Laura!

E: Y en los no-vivos... ¿no hay células?

P3: ¿Quién pregunta? ¿Qué hay?, ¿qué llega a haber?, ¿cuál es la organización más compleja?

[Otros estudiantes tratan de contestar.]

P3: Paren, dejen pensar a quien le acabo de preguntar. [Al estudiante.] Olvidate que estoy yo, decí lo que pensás.

Todos los que participan en la clase son simultáneamente emisores y receptores; se hace uso frecuente de la realimentación para reconocer la comprensión correcta:

P3: Para hacer esa comida, ¿qué necesita? 
E: Agua, sal, tierra, tallos, luz... P3: Agua, sal... ¿Conocés la partes de la planta?

E: Tallo, sépalo, pétalo.

P3: De las partes de la planta, ¿hay alguna que no reciba luz?

E: La raíz.

P3: Una célula de la raíz, ¿es una célula vegetal? Todo eso lo vamos a ir revisando en varias clases.

En estas clases apareció un fuerte compromiso emocional de la profesora P3 transmitido a sus estudiantes. Los diálogos transcritos denotan interés, confianza, respeto, tolerancia y disposición a escuchar; se establece entonces lo que Burbules (1999) considera una genuina comunicación pedagógica, de carácter dialógico e interactivo (Mortimer y Scott, 2003).

Las preguntas auténticas (que quedan fundamentalmente abiertas, ya que la profesora no preestablece la respuesta) son las que más aparecen en los diálogos que observamos:

E: Me contaron que los enfermos del [mal de] Chagas deben tomar mucha leche. P3: ¡Cuántas cosa raras que me contaron! [Se refiere también al comentario previo de otra estudiante sobre que, para evaluar si su sobrino era portador de la enfermedad, lo hacían picar por vinchucas sanas.] Es otra cosa que tengo que averiguar para la próxima.

Otra E: Los chagásicos toman muchos antibióticos. Capaz que por eso toman mucha leche.

P3: Tenés razón, para recomponer la flora intestinal. ¡Muy bien, señora! ¡Cómo razona!

La profesora $\mathrm{P} 3$ permanentemente da oportunidades para que los estudiantes puedan aprender a hablar, escribir y pensar acerca de los modelos científicos (Izquierdo y Adúriz-Bravo, 2003; Izquierdo et ál., 2004). Según Lemke (1997, 2002), capacitar a los estudiantes en el uso del lenguaje especializado de la ciencia es esencial para alcanzar los fines de la educación científica, preparándolos para usar la ciencia en aquellos actos que directamente afecten sus propios intereses. Esta profesora, a través de actividades como la redacción de monografías y el análisis de artículos periodísticos, permite que los estudiantes aprendan a hablar y escribir en forma más rica y compleja sobre asuntos sociocientíficos, trabajando las ideas previas que ellos traen y discutiendo los modelos del sentido común sobre esos temas. En clase se analiza la manera en que se habla de ellos desde el sentido común y desde la ciencia.

La profesora P3 hizo hincapié en la comprensión de los términos científicos que la clase utilizaba, dándoles un sentido, evitando el uso excesivo de los mismos ("Levanten la mano a los que les suene chino", "Aquí largaron con las palabras del ahorcado"). También enfatizó el carácter de la ciencia como actividad humana en la discusión que se suscitó en la clase acerca de la película Casas de fuego, reflexionando sobre las trabas que las sociedades científicas ponen a sus miembros cuando ellos se enfrentan al poder hegemónico.

Todos estos recursos contribuyen a una construcción activa del conocimiento por parte de los estudiantes (Bransford y Vye, 1989). Decimos por ello que el modelo "didáctico" en el que se inscribe la profesora es constructivista: considera al estudiante como un 
agente activo que construye significados científicos escolares en respuesta a la situación educativa. En ningún momento la profesora P3 trabaja con el supuesto de que sus estudiantes no operan con la información suministrada por ella misma o por los libros. No se castiga o evita el error, sino que se busca usarlo como fuente de aprendizaje (Astolfi, 1997).

\section{Casos 4 y 5}

Las observaciones C7 y C8 se realizaron en una cuarta institución (ver tabla 1), en dos clases dictadas por distintas profesoras y con distintos grupos de estudiantes. La clase C7 consistió en la proyección de un video que integraba los conocimientos de mitosis y meiosis. En primera instancia la profesora $\mathrm{P} 4$ hizo un pequeño repaso del proceso de mitosis con el objetivo de discutir y resaltar distintos aspectos sobre el proceso, que aparecían en el video. Para terminar la clase, esquematizaron en conjunto el proceso de meiosis, resaltaron las diferencias con la mitosis y ubicaron ambos procesos en las células y órganos donde se producen en distintos organismos.

La estructura de la clase fue un diálogo guiado por la profesora, cuyo objetivo parecía ser el de integrar los conocimientos adquiridos. Los estudiantes contestaban generalmente en forma correcta a las preguntas de la profesora, y aunque algunas veces ella tomaba la respuesta incorrecta, en general remarcaba la respuesta correcta, que usaba como base para una nueva pregunta. Cada tanto estimulaba a los estudiantes a sostener la secuencia dialógica, con frases como: "No se distraigan", "No abandones", "Perdés la ilación de lo que hablamos".
En esta observación, la profesora P4 pareció inscribirse con bastante coherencia en un modelo conductista (Astolfi, 1997), tal vez elegido por ella porque se adaptaba mejor a los objetivos de refuerzo de su clase, que eran integrar y repasar los conocimientos acerca de temas tratados en clases anteriores. Desde una perspectiva conductista, el estudiante aprende mediante una serie de condicionamientos: los diálogos muestran que la profesora busca todo el tiempo la asociación entre estímulo, respuesta y refuerzo de las conductas deseadas. Se infiere la búsqueda de una pedagogía del éxito, puesto que la profesora trata de encontrar los medios para evitar el error privilegiando las respuestas correctas (“¡Exactamente!!!”) y desconociendo las incorrectas. El modelo conductista suele aplicarse para afianzar y evaluar los logros alcanzados en el aprendizaje de un tema; esto puede explicar por qué la profesora lo eligió para su clase.

Las preguntas son predominantemente aparentes (pseudopreguntas):

P4: Previo a la división celular mitótica, ¿qué ocurre con los cromosomas?

E: Se duplican.

P4: Por lo tanto son cromosomas...

E: ... bivalentes...

P4: ... porque tienen el doble de información genética.

P4: [Señalando una etapa de la meiosis.] Por lo que observan, ¿de qué etapa se trata?

E: Metafase.

Otros Es: Profase.

P4: Algunos dijeron metafase; todavía no nos mostraron los cromosomas en la placa ecuatorial. ¿Qué ocurre en la profase? 
E: Puede haber intercambio de información.

P4: En esto nos detenemos. Puede haber entrecruzamiento de material genético, que nos explica....

E: ... la variación...

P4: ... la variación que encontramos en las gametas. Puede haber entrecruzamiento de cromosomas porque como dijimos se ocupan...

E: ... de un mismo carácter.

Algunos profesores, cuando hacen preguntas a sus estudiantes, tienden a ajustarse al clásico modelo de iniciaciónrespuesta-evaluación (IRE). Esto se ve muy claro en el diálogo anterior, en la secuencia "nos explica (I)... la variación (R)... en las gametas (E)", que muestra un intento de co-construcción dialógica forzada. Las pseudopreguntas sirven para mantener una tosca apariencia de un aula orientada hacia la discusión, pero denotan el deseo de la profesora P4 de mantener un verdadero control de la comunicación. En este marco rara vez los estudiantes cuestionan el sentido de lo que se dice o introducen preguntas que salven el abismo entre su marco de referencia y el de la ciencia escolar presentada por la profesora; se produce entonces un severo vaciamiento de significaciones científicas (Galagovsky et ál., 1998).

Por otra parte, las pseudopreguntas le sirven a la profesora P4 para guiar a los estudiantes en los sucesivos pasos de un razonamiento complejo (Burbules, 1999), y en este sentido encuentran su utilidad como "andamios", desde la perspectiva vigotskiana. Este sería un caso interesante en el cual una comunicación relativamente lineal (en modelo orquesta restringido) permite sin embar- go el despliegue de estrategias de cariz constructivista.

En una segunda clase (C8), dada por otra profesora (P5), que observamos en esta misma institución, se trabajó con mezclas heterogéneas y homogéneas, como parte del estudio de la composición química de un río que surcaba una ciudad ficticia que los estudiantes habían creado. A lo largo de todo el año la clase trabaja sobre esta ciudad situándose en distintos roles: científicos, legisladores, pobladores, etc. Se comenzó con el sistema urbano, analizando el agua en él. Durante la clase observada los estudiantes trabajaron con mezclas heterogéneas y homogéneas del río, construyeron en grupos modelos de soluciones con masillas de distintos colores, que representaban los distintos átomos.

La profesora P5 inició el nuevo tema pidiendo ejemplos de mezclas homogéneas, y luego entre todos acordaron una definición sobre ambos tipos de mezclas y discutieron los factores que influyen en la solubilidad. Los estudiantes trabajaron solos y la profesora circulaba entre las mesadas; los estudiantes consultaban distendidamente y se los veía comprometidos con la tarea. Había un ambiente amable y participativo.

Decidimos considerar que la profesora P5, al igual que la profesora P3, sostiene una práctica que se inscribe en su mayoría en un modelo constructivista: toma el error como fuente de aprendizaje, usa preguntas abiertas, considera esencial la participación cognitiva y lingüística de los estudiantes. El modelo de comunicación que parece establecerse es sistémico durante buena parte del tiempo de tarea. Esto se ve en: 
1. El uso de modelos y analogías (P5 usa una analogía para que los estudiantes entiendan el modelo de solución).

2. El establecimiento de puentes entre el lenguaje coloquial y científico:

P5: Vamos a hacer otros modelos pero no con masilla, para que no se saturen. En lenguaje cotidiano "saturarse" quiere decir estar harto, cansado. Pero las soluciones no se cansan.

3. La aplicación de los contenidos científicos en contextos cotidianos, que apunta a una enseñanza de las ciencias ligada a la comprensión:

P5: En la cocina hacemos mezclas homogéneas ¿Cuáles?

Es: Jugo, agua con azúcar, agua con sal...

Perkins (1995: 40) propone una enseñanza basada en la retención, la comprensión y el uso activo del conocimiento, ya que "las investigaciones indican que los alumnos aprenden más cuando organizan los hechos, los relacionan con el conocimiento anterior, utilizan asociaciones visuales, se examinan a sí mismos y elaboran y extrapolan lo que están leyendo o escuchando". Bransford y Vye (1989), por su parte, sostienen que el conocimiento debe ser construido activamente por los estudiantes, pues si ellos no tienen la posibilidad de usar la nueva información para lograr objetivos específicos, a menudo sólo recuerdan los contenidos científicos en contextos restringidos, y estos no soportan la aplicación ni la transferencia. Todo ello se manifiesta hasta cierto punto en el uso del diálogo genuino que hace esta profesora.

\section{Conclusión}

Este trabajo representa un acercamiento modesto al análisis de la comunicación en el aula de ciencias naturales y su relación con las formas de enseñanza declaradas y efectuadas. Dentro del recorte que implican nuestras observaciones, a partir del análisis de las clases observadas surgen las siguientes apreciaciones:

1. En las clases observadas en las cuales se reconocen muchos elementos del modelo constructivista de enseñanza (C5, C6, C8 y C9), el esquema de comunicación que se establece en el aula se ajusta gran parte del tiempo a un modelo de tipo sistémico. En este sentido, las profesoras (P3 y P5) que adhieren genuinamente (y no sólo desde la declaración) al constructivismo son capaces de generar espacios de comunicación dialógica que permitan la construcción, negociación y el compartir de significados. En estas clases, aparece un fuerte compromiso emocional por parte de las profesoras, que sus estudiantes se apropiaban provechosamente. Las preguntas auténticas, de indagación (Duschl, 1998), eran las que más aparecían en los diálogos que se establecían entre profesoras y estudiantes.

2. En las clases observadas en las cuales las profesoras, contrariando sus propios dichos, recreaban modelos de enseñanza por transmisión o por condicionamiento (clases C1, C2, C3, C4 y C7), la comunicación seguía un esquema lineal de emisor a receptor (Astolfi, 1997), representable mediante el modelo telegráfico (Contreras, 1990). También emergía a veces un modelo orquestal bastante res- 
trictivo, con secuencias IRE: en estas clases aparecían casi únicamente preguntas convergentes que se dirigían a una respuesta específica, típicas de los modelos de enseñanza tradicionales. Las pseudopreguntas usadas comunican mensajes implícitos con las respuestas deseables por el docente; se apoyan en la ingenuidad comunicativa de los alumnos, que no desenmascaran el vaciamiento del lenguaje científico. En estas clases, los estudiantes rara vez cuestionaron el significado de lo que se estaba enunciando o introdujeron participaciones para salvar el abismo entre su marco de referencia y el de las profesoras. La preocupación por el control de la situación empobreció el diálogo.

3. Algunas profesoras que manifiestan adherir a un modelo constructivista no reconocen en el error una fuente de aprendizajes científicos. Si el error es negado o ignorado, el diálogo necesariamente se empobrece, ya que la libertad que tienen los estudiantes para plantear sus dudas es limitada, y el diálogo se limita a secuencias más o menos establecidas. El acercamiento de las profesoras P3 y P5 al modelo constructivista, que intentó tomar el error como fuente de aprendizaje sin castigarlo ni evitarlo, permitió que se estableciera un diálogo abierto en el que los estudiantes participaron con más libertad, con mayor grado de compromiso, mayor motivación y más creatividad.

4. Acordamos con Lemke (1997) en que un diálogo didáctico abierto permitió a las profesoras P3 y P5 desarrollar oportunidades para:
- Discutir las teorías de sentido común sobre ciertos temas y relacionarlos con la manera en que se habla de ellos desde la ciencia.

- Tender puentes entre el lenguaje coloquial y el científico.

- Preparar a los estudiantes para usar la ciencia en la fundamentación de situaciones cotidianas.

- Enfatizar el carácter de la ciencia como actividad humana.

Otro punto de importancia es que, cuando se dan en el profesorado de ciencias concepciones teóricas inhomogéneas de la tarea de enseñar (Porlán y Rivero, 1998, 2001), la adherencia a determinados modelos de enseñanza puede depender fuertemente de factores contextuales, algunos de los cuales señalamos en el análisis de datos: la finalidad de la clase, el ethos y el clima institucional, el apoyo de los directivos, los materiales de aula que se están trabajando.

En nuestra opinión, el establecimiento de un esquema más sistémico para la comunicación dentro de un aula de ciencias naturales con enfoque genuinamente constructivista favorecería una reconstrucción racional y razonada (Izquierdo et al., 2004) del conocimiento científico por parte de los estudiantes. Permitiría que los estudiantes piensen el mundo natural valiéndose del conocimiento teórico que aprenden, lo que constituye el núcleo del llamado modelo cognitivo de ciencia escolar (Izquierdo et ál., 1999; Izquierdo y Adúriz-Bravo, 2003). En este sentido, usos de la comunicación más obturadores o clausurantes, tales como el habla de control, las pseudopreguntas con selección de las respuestas "esperadas", la nomi- 
nalización que "vacía” contenidos, o la ironía usada como elemento de poder, no vehiculan adecuadamente las es-

\section{Referencias}

Adúriz-Bravo, A. (2001). Integración de la epistemología en la formación del profesorado de ciencias. Tesis doctoral. Bellaterra: UAB. [En línea.]

Apple, M. (1979). Ideology and curriculum. Londres: Routledge \& Kegan Paul.

Astolfi, J.-P. (1997). Aprender en la escuela. Santiago de Chile: Dolmen.

Ausubel, D., Novak, J. y Hanesian, H. (1983). Psicología educacional: un punto de vista cognoscitivo. México: Trillas.

Bransford, J. y Vye, N. (1989). A perspective on cognitive research and its implications for instruction. En Resnick, L. y Klopfer, L. (eds.). Toward the thinking curriculum: Current cognitive research, 173-205. Alexandria: Association for Supervision and $\mathrm{Cu}$ rriculum Development.

Burbules, N. (1999). El diálogo en la enseñanza: teórica y práctica. Buenos Aires: Amorrortu.

Camilloni, A. (1998). Las apreciaciones personales del profesor. Publicación interna de la Cátedra de Didáctica I de la Facultad de Filosofía y Letras. Buenos Aires: Universidad de Buenos Aires.

Cañal, P. y Porlán, R. (1987). Una experiencia de aprendizaje por investigación directa del medio en la formación de maestros. Revista de Educación, 284, 273-294. trategias didácticas propuestas por el constructivismo. $\mathbf{\Delta}$

Contreras, J. (1990). Enseñanza, currículum y profesorado. Madrid: Akal.

Develaki, M. (2007). The model-based view of scientific theories and the structuring of school science programmes. Science \& Education, 16(7), 725-749.

Duschl, R. (1998). La valoración de argumentaciones y explicaciones: Promover estrategias de retroalimentación. Enseñanza de las Ciencias, 16(1), 3-20.

Erduran, S. y Duschl, R. (2004). Interdisciplinary characterizations of models and the nature of chemical knowledge in the classroom. Studies in Science Education, 40, 111-144.

Galagovsky, L. y Adúriz-Bravo, A. (2001). Modelos y analogías en la enseñanza de las ciencias naturales: El concepto de modelo didáctico analógico. Enseñanza de las Ciencias, 19(2), 231-242.

Galagovsky, L., Bonan, L. y Adúriz-Bravo, A. (1998). Problemas con el lenguaje científico en la escuela. Un análisis desde la observación de clases de ciencias naturales. Enseñanza de las ciencias, 16(2), 315-321.

Gallego Badillo, R. (2004). Un concepto epistemológico de modelo para la didáctica de las ciencias experimentales. Revista Electrónica de Enseñanza de las Ciencias, 3(3). [En línea.] 
Izquierdo, M. (2005). Las estructuras retóricas de los libros de texto. Tarbiya, 36, 11-34.

Izquierdo, M. y Adúriz-Bravo, A. (2003). Epistemological foundations of school science. Science \& Education, 12(1), 27-43.

Izquierdo, M. y Aliberas, J., con la colaboración de Adúriz-Bravo, A. (2004). Pensar, actuar i parlar a la classe de ciències: Per un ensenyament racional i raonable. Bellaterra: Servei de Publicacions de la UAB.

Izquierdo, M., Espinet, M., García, M. P., Pujol, R.M. y Sanmartí, N. (1999). Caracterización y fundamentación de la ciencia escolar. Enseñanza de las Ciencias, número extra, 79-91.

Izquierdo, M., Marzábal, A., Márquez, C. y Gouvea, G. (2007). Experimental stories in science textbooks. Paper presentado en la 7th ESERA (European Science Education Research Association) International Conference, Malmö, Suecia.

Jiménez Aleixandre, M. P. (1996). Dubidar para aprender. Vigo: Edicións Xerais de Galicia.

Lemke, J. (1997). Aprender a hablar ciencia. Barcelona: Paidós.

Lemke, J. (2002). Enseñar todos los lenguajes de la ciencia: Palabras, símbolos, imágenes y acciones En Benlloch, M. (ed.). La educación en ciencias: Ideas para mejorar su práctica, 159-186. Barcelona: Paidós.

Levin, L., Ramos, A. M. y Adúriz-Bravo, A. (2004). Relaciones entre el modelo didáctico al que adhieren los profesores de ciencias naturales y el modelo comunicativo que establecen en sus aulas. Paper presentado en el I Congreso de Educación en Ciencia y Tecnología, San Fernando del Valle de Catamarca, Argentina.
Marbà, A. y Márquez, C. (2006). Learning to read biology (and reading to learn biology). Paper presentado en la ERIDOB (European Researchers in Didactics of Biology) 2006 International Conference, Londres, Reino Unido.

Marbà, A., Márquez, C. y Prat, À. (2006). La lectura en el proceso de aprendizaje de los modelos científicos. En Quintanilla, M. y Adúriz-Bravo, A. (eds.). Enseñar ciencias en el nuevo milenio: Retos y propuestas. Santiago de Chile: Ediciones de la PUC.

Márquez, C., Izquierdo, M. y Espinet, M. (2003). Comunicación multimodal en la clase de ciencias: el ciclo del agua. Enseñanza de las Ciencias, 21(3), 371-386.

Márquez, C.; Izquierdo, M. y Espinet, M. (2006). Multimodal science teachers' discourse in modelling the water cycle. Science Education, 90(2), 202226.

Mortimer, E. y Scott, P. (2003). Meaning making in secondary science classrooms. Buckingham: Open University Press.

Perkins, D. (1995). La escuela inteligente. Barcelona: Gedisa.

Porlán, R. y Rivero, A. (1998). El conocimiento de los profesores. Sevilla: Díada.

Porlán, R. y Rivero, A. (2001). Nature et organisation du savoir professionnel enseignant «souhaitable». Aster, 32, 221-251.

Rice, S. (2005). Teaching and learning through story and dialogue. Educational Theory, 43(1), 85-97.

Sagastizábal, M. Á. (coord.) (2006). Aprender y enseñar en contextos complejos. Buenos Aires: Novedades Educativas. 
Sanmartí, N. (2002). Didáctica de las ciencias en la educación secundaria obligatoria. Madrid: Síntesis.

Sanmartí, N. (coord.) (2003). Aprendre ciències tot aprenent a escriure ciència. Barcelona: Edicions 62.

Sarramona, J. (ed.) (1988). Comunicación y educación. Barcelona: CEAC.

Scott, P. y Ametller, J. (2007). Teaching science in a meaningful way: Striking a balance between 'opening up' and 'closing down' classroom talk. School Science Review, 88(324), 77-83.
Scott, P., Mortimer, E. y Aguiar, O. (2006). The tension between authoritative and dialogic discourse: A fundamental characteristic of meaning making interactions in high school science lessons. Science Education, 90(4), 605-631.

Sierra, F. (2000). Introducción a la teoría de la comunicación educativa. Sevilla: MAD.

Watzlawick, P., Beavin, J. H. y Jackson, D. D. (1981). Teoría de la comunicación humana. Interacciones, patologías y paradojas. Barcelona: Herder. (Edición original en inglés de 1967). 